\title{
The influence of a formula supplemented with dairy lipids and plant oils on the erythrocyte membrane omega-3 fatty acid profile in healthy full-term infants: a double-blind randomized controlled trial
}

\author{
Maria Lorella Giannì ${ }^{*}$, Paola Roggero ${ }^{1}$, Charlotte Baudry², Amandine Ligneul², Daniela Morniroli', \\ Francesca Garbarino', Pascale le Ruyet ${ }^{2}$ and Fabio Mosca ${ }^{1}$
}

\begin{abstract}
Background: Human milk is the optimal nutrition for infants. When breastfeeding is not possible, supplementation of infant formula with long chain polyunsaturated fatty acids appears to promote neurodevelopmental outcome and visual function. Plant oils, that are the only source of fat in most of infant formulas, do not contain specific fatty acids that are present in human and cow milk and do not encounter milk fat triglyceride structure. Experimental data suggest that a mix of dairy lipids and plant oils can potentiate endogenous synthesis of n-3 long chain polyunsaturated fatty acids. This trial aims to determine the effect of an infant formula supplemented with a mixture of dairy lipids and plant oils on the erythrocyte membrane omega-3 fatty acid profile in full-term infants (primary outcome). Erythrocyte membrane long chain polyunsaturated fatty acids and fatty acids content, the plasma lipid profile and the insulin-growth factor 1 level, the gastrointestinal tolerance, the changes throughout the study in blood fatty acids content, in growth and body composition are evaluated as secondary outcomes.

Methods/Design: In a double-blind controlled randomized trial, 75 healthy full-term infants are randomly allocated to receive for four months a formula supplemented with a mixture of dairy lipids and plant oils or a formula containing only plant oils or a formula containing plant oils supplemented with arachidonic acid and docosahexaenoic acid. Twenty-five breast-fed infants constitute the reference group. Erythrocyte membrane omega-3 fatty acid profile, long chain polyunsaturated fatty acids and the other fatty acids content, the plasma lipid profile and the insulin-growth factor 1 level are measured after four months of intervention. Gastrointestinal tolerance, the changes in blood fatty acids content, in growth and body composition, assessed by means of an air displacement plethysmography system, are also evaluated throughout the study.

(Continued on next page)
\end{abstract}

\footnotetext{
* Correspondence: maria.gianni@unimi.it

${ }^{1}$ Neonatal Intensive Care Unit (NICU), Department of Clinical Science and Comunity Health, Fondazione IRCCS "Ca' Granda" Ospedale Maggiore Policlinico, University of Milan, Milan, Italy

Full list of author information is available at the end of the article
} 
(Continued from previous page)

Discussion: The achievement of an appropriate long chain polyunsaturated fatty acids status represents an important goal in neonatal nutrition. Gaining further insight in the effects of the supplementation of a formula with dairy lipids and plant oils in healthy full-term infants could help to produce a formula whose fat content, composition and structure is more similar to human milk.

Trial registration: ClinicalTrials.gov Identifier NCT01611649

Keywords: Full-term infants, Formula supplementation, Dairy lipids, Erythrocyte membrane omega-3 fatty acid profile

\section{Background}

Human milk is recognized as the gold standard for infant nutrition [1-3]. Lipids are a major component of human milk and provide $45 \%$ of total energy intake. The main constituents are triacylglycerols, phospholipids and their components fatty acids and sterols. Lipids contribute to several biological functions with regard to growth and development. In particular, human milk provides essential fatty acids (EFA) and long chain polyunsaturated fatty acids (LC-PUFA) of omega- 3 and omega- 6 families, such as docosahexaenoic acid (DHA, 22:6n-3) and arachidonic acid (AA, 20:4n-6) [4]. These fatty acids constitute the main components of brain tissue and have an important impact on neuronal and visual functions [5]. LC-PUFA accretion in human brain is a slow gradual process [6]. The last trimester of pregnancy represents a critical period for LC-PUFA deposition whose accretion in the nervous system endures after birth [7-9]. The deposit of AA during the last trimester of pregnancy differs from that of DHA so that at term human brain contains relatively more AA than DHA. However, after term, DHA brain content increases, resulting in DHA being the main LC-PUFA in adult brain. It has been demonstrated that lipids represent 50 to $60 \%$ of dry adult brain weight [10]. In addition, cerebral LC-PUFA concentration seems to be higher in the cortical gray matter and lower in the white matter $[11,12]$. In baboon neonates, DHA and AA have been found to be present at highest concentrations in the precentral, postcentral, prefrontal and occipital cortices, and also in basal ganglia, hippocampus, thalamus and cerebellum [12]. As these areas are mainly involved in the development of sensor-motor integration, attention and memory functions, these data suggest the critical role of LC-PUFA in promoting these skills.

Experimental studies confirmed that a deficient dietary intake of DHA can lead to a reduced neuronal cell size, a decreased visual function and a compromised learning behavior [13,14].

DHA and AA can be directly provided from the diet or be synthesized from endogenous conversion of the precursors alpha-linolenic acid (ALA; 18:3 $\omega 3$ ) and linoleic acid (LA; $18: 2 \omega 6)$ by enzymatic processes, which are present also in fetuses and infants. However, these enzymatic systems seem to be unable to satisfy LC-PUFA requirements in infants until 16 weeks after birth $[15,16]$. Accordingly, LC-PUFA during pregnancy are mainly supplied by the placental transfer, whereas during the first months of life the infant is dependent on LCPUFA supply through breast milk or formula [17].

Fatty acid status can be evaluated by measuring the fatty acid composition of functional tissue such as brain or retina. However, in clinical studies, substitute parameters can be used. It has been demonstrated that erythrocyte membrane fatty acids are representative of brain cell membranes composition, whereas serum lipid levels are influenced by other transient factors, such as contingent diet $[18,19]$. Therefore, DHA levels in erythrocyte membrane phospholipids are commonly used as an indicator of brain DHA status.

When breastfeeding is not possible, milk substitutes represent the nutritional alternative. It has been demonstrated that formula fed infants have lower levels of LCPUFA in their cerebral cortex than breastfed infants. This relative deficit of LC-PUFA may partially explain the lower Intelligence Quotient scores reported in formula fed infants in comparison with breastfed infants [20,21]. Makrides M. et al. [22] reported a positive correlation between the erythrocyte DHA levels and the visual-evoked potential acuity. In addition, the authors demonstrated that full-term formula fed infants had erythrocyte DHA levels lower than breast fed infants [22]. Farquharson J. et al. [23] have found lower levels of LC-PUFA in the cerebral cortex of infants fed a formula enriched only with EFA than in breastfed infants. These results suggest that formulas containing only ALA and LA may not be adequate to satisfy the actual requirements of infants in terms of LC-PUFA. Furthermore, it has been reported that infants fed a formula supplemented with DHA and AA have higher erythrocyte membrane omega-3 concentrations at 9 months of age as compared to infants fed an unsupplemented formula [24]. Indeed, supplementation of infant formula with LC-PUFA appears to be associated with a beneficial effect on short-term neurodevelopmental outcome and visual function [25-27]. Although evidence concerning 
the persistence of the beneficial effect beyond the fourth month of age is lacking, it cannot be excluded that the positive effect of LC-PUFA supplementation may become again evident at school age when infants are required to perform tasks that necessitate more complex neural functions [15].

Besides, DHA consumption appears to influence infants' body composition by promoting the development of fat free mass without any detrimental effect on growth [27]. Courville AB. et al. [28] have recently demonstrated that infants of mothers consuming food supplemented with DHA during the last half of pregnancy have lower ponderal indices and umbilical cord blood insulin concentrations than infants of mothers consuming the placebo.

Most of infant formulas available nowadays on the market contain plant oils as the only source of fat [29]. Indeed, infant formulas have been enriched in EFA-rich plant oils as cow milk fat does not contain enough EFA to meet infant's needs [30]. The main plant oils used are coconut oil, corn oil, soybean oil, palm olein, palm kernel oil, palm oil, high oleic safflower oil, peanut oil, and, in Europe, low-erucic acid rapeseed oil. Vegetable oilbased formulas can contain up to $4 \%$ residual milk fat [31]. However, plant oils do not contain specific fatty acids, particularly short chain fatty acids, that are present in human and cow milk [32,33] and constitute a pertinent energy source for infants [31]. In addition, plant oils do not encounter milk fat triglyceride structure [30].

The supplementation of infant formulas with dairy lipids could provide a fat composition and structure closer to human milk, thus improving the quality of formula fat composition. Dabadie et al. [34] demonstrated that dairy lipids associated with rapeseed oil significantly increased erythrocyte DHA levels adults. Recently, it has been reported that rodents consuming a diet with a mix of dairy lipids and plant oils showed levels of brain DHA higher than rodents consuming a diet containing only plant oils or a DHA-enriched diet containing plant oils, with the same ALA contents [35]. These data suggest that a mix of dairy lipids and ALA-rich plant oils could potentiate endogenous n-3 LC-PUFA synthesis.

\section{Study aims}

\section{Primary aim}

To investigate the effect of an infant formula supplemented with a mixture of dairy lipids and plant oils (formula A) on the erythrocyte membrane omega-3 fatty acid profile in healthy full-term infants as compared to a formula containing only vegetable lipids (formula $\mathrm{B}$ ) or vegetable lipids supplemented with LC-PUFA (AA + DHA) (formula C).

\section{Secondary aims}

1) To compare erythrocyte membrane LC-PUFA content of infants consuming formula $\mathrm{A}$ in comparison to breastfed infants (reference group).

2) To compare the changes throughout the study in blood fatty acids content exhibited by infants consuming formula $\mathrm{A}$ in comparison to infants consuming formula $\mathrm{B}$ and formula $\mathrm{C}$ and to breastfed infants (reference group).

3) To compare the plasma lipid profile and the insulingrowth factor 1 (IGF-1) levels exhibited by infants consuming formula $\mathrm{A}$ in comparison to infants consuming formula $\mathrm{B}$ and formula $\mathrm{C}$ and to breastfed infants (reference group).

4) To investigate the gastrointestinal tolerance of formula A.

5) To evaluate the growth and the body composition changes exhibited by infants consuming formula $\mathrm{A}$ in comparison to infants consuming formula $\mathrm{B}$ and formula $\mathrm{C}$ and to breastfed infants (reference group).

6) To compare the erythrocyte membrane fatty acid profile exhibited by infants consuming formula $\mathrm{A}$ in comparison to infants consuming formula $\mathrm{B}$ and formula $\mathrm{C}$ and to breastfed infants (reference group).

\section{Methods and design}

The study is designed as a mono-centric, double-blind, randomized controlled trial. The Ethical Committee of the Fondazione IRCCS Cà Granda Ospedale Maggiore Policlinico, University of Milan, Italy, approves the study protocol before the start of the study. Written informed consent is obtained from both parents.

\section{Study population}

All infants who are born in the Department of Neonatology of the Fondazione IRCCS Cà Granda Ospedale Maggiore Policlinico, University of Milan, are screened for participation in the study.

Inclusion criteria are: gestational age 37 to 42 weeks, birth weight $>2500 \mathrm{~g}$, healthy newborns from normal pregnancy, aged up to 3 weeks when entering the study. Exclusion criteria are: newborns whose parents have planned to move within 6 months after birth, newborns with a positive family history of allergy to milk proteins, newborns with known congenital or postnatal diseases which could interfere with the study. With regard to the reference group, additional inclusion criteria were the intention of mothers to exclusively breastfeed their infants at least for 4 months and being exclusively breastfed at time of enrollment. 


\section{Subject enrollment and randomization}

Mothers are encouraged to breastfeed their newborn for at least 4 months. Only if mothers cannot or intend not to breastfeed their newborns, the study team asks the parents for their consent to participate in the study and to be randomized to one of the three formula groups. Enrollment and randomization occur at the same time and are performed within 21 days after delivery.

Infants whose mothers indicate that they intend to exclusively breastfeed are included in the breastfeeding group. This group serves as the non-randomized reference group.

The randomization schedule (provided by Lactalis Recherche et Developpement, Nutrition Department, Retiers, France) is computer-generated and stratified on sex. Sequentially numbered tins of infant formula are prepared according to this schedule. All formulas are packaged in blinded containers labeled only with the study details and the number of randomization; they are indistinguishable in appearance and texture. Once the newborn is enrolled, he/she is allocated to the next available study number which corresponds to the allocation to one of the 3 study formulas. Both the investigators and the infants' parents are blind to the group allocation.

\section{Interventions}

At enrollment, each newborn is allocated to one of the three formula groups. Newborns randomized to formula A receive a formula containing a mixture of dairy lipids and plant oils; newborns randomized to formula B receive a formula containing only plant oils; newborns randomized to formula $C$ receive a formula containing plant oils supplemented with AA and DHA. Compositions of the three formulas are detailed in Table 1. Study formulas are started straightaway after randomization and are provided for the four subsequent months. The study formulas are formulated into powders and are

Table 1 Composition of the infant formulas

\begin{tabular}{lcccc}
\hline & & Formula A & Formula B & Formula C \\
\hline \multirow{2}{*}{ Energy } & $\mathrm{kJ}$ & $100 \mathrm{ml}$ & $100 \mathrm{ml}^{*}$ & $100 \mathrm{~m}^{*}$ \\
& $\mathrm{kcal}$ & 66 & 275 & 275 \\
Protein & $\mathrm{G}$ & 1.3 & 66 & 66 \\
Carbohydrates & $\mathrm{G}$ & 8.1 & 1.3 & 1.3 \\
Lactose & $\mathrm{G}$ & 6.8 & 8.1 & 8.1 \\
Fat & $\mathrm{G}$ & 3.1 & 6.8 & 6.8 \\
Linoleic acid & $\mathrm{mg}$ & 439 & 3.1 & 3.1 \\
alpha linolenic acid & $\mathrm{mg}$ & 73 & 549 & 549 \\
AA & $\mathrm{mg}$ & & 55 & 55 \\
DHA & $\mathrm{mg}$ & & & 12.4 \\
\hline
\end{tabular}

* Reconstituted $13.3 \%$. reconstituted at $13.3 \%$. All study formulas are currently on the market; they are manufactured and provided by Lactalis, Craon, France in compliance with the European directive on infant formulae.

Infants are fed on demand and are not allowed to introduce complementary foods while receiving one of the study formulas.

\section{Study outcome measures}

The study schedule is reported in Table 2 .

\section{Measurement of erythrocyte membrane and blood fatty acid levels, plasma lipid profile (triglycerides, high density lipoprotein, low density lipoprotein, total cholesterol) and IGF-1 levels}

Venous blood samples are drawn after consumption of the allocated formula for 4 months. The blood is collected on heparin. Plasma is separated by 15 -min centrifugation $\left(2200 \mathrm{~g}\right.$ at $\left.4^{\circ} \mathrm{C}\right)$ from erythrocytes that are rinsed with saline solution $(\mathrm{NaCl} 0.9 \%)$. The plasma and erythrocytes are stored at $-80^{\circ} \mathrm{C}$ for later analysis.

Erythrocyte membrane FA levels are measured by means of a gas-liquid chromatography (HPLC) by ITERG, Pessac, France. Sum of omega-3 FA levels includes ALA, EPA (eicosapentaenoic acid, 20:5n-3), DPA (docosapentaenoic acid, 22:5n-3) and DHA levels. Plasma lipid profile and IGF1 levels are measured by means of standard techniques.

\section{Measurement of blood FA levels}

Whole blood samples are collected using a heel stick at enrollment and after four months of consumption of the

Table 2 Study schedule

\begin{tabular}{|c|c|c|c|c|c|}
\hline & V1 & V2 & V3 & V4 & V5 \\
\hline Written informed consent & $x$ & & & & \\
\hline Personal and family history & $x$ & & & & \\
\hline Inclusion and exclusion criteria & $x$ & & & & \\
\hline Randomization & $x$ & & & & \\
\hline Phone calls & & $x$ & & $x$ & \\
\hline Heel stick blood sampling & $x$ & & & & $x$ \\
\hline Venipuncture & & & & & $x$ \\
\hline $\begin{array}{l}\text { Anthropometric parameters } \\
\text { (weight, length, head circumference) }\end{array}$ & $x$ & & $x$ & & $x$ \\
\hline Body composition & $x$ & & $x$ & & $x$ \\
\hline $\begin{array}{l}\text { Evaluation of onset and severity of } \\
\text { atopic dermatitis by means } \\
\text { of the SCORAD index }\end{array}$ & $x$ & & $x$ & & $x$ \\
\hline Adverse events & \multicolumn{5}{|c|}{ Throughout the study } \\
\hline
\end{tabular}


allocated formula. FA levels are quantified by means of a gas-liquid chromatography (HPLC) by Oxigenlab, Brescia, Italy.

\section{Investigation of the gastrointestinal tolerance}

Parents are contacted every four weeks either by clinic visits or phone calls. Specifically, the following indicators of gastrointestinal tolerance are collected after one and three months of consumption of the allocated formula: amount of formula consumed, regurgitation/reflux, colic (intermittent attacks of abdominal pain when the baby screams and draws up his/her legs but is well between episodes), daily frequency of stool passage, stool consistency and color. In addition, during the clinic visits, the onset, if present, and the severity of atopic dermatitis is evaluated by means of the SCORAD index [36].

\section{Growth and body composition}

Birth weight, length and head circumference are recorded. Growth and body composition are then evaluated at enrollment, after 2 and 4 months of consumption of the allocated formula. The infants' anthropometric measurements (body weight, length, head circumference) are obtained using standardized techniques [37]. Subject mass is measured on an electronic scale accurate to the nearest $0.1 \mathrm{~g}$. Recumbent length is measured on a Harpenden stadiometer to the nearest $1 \mathrm{~mm}$. The head circumference is measured using a non-stretch measuring tape to the nearest $1 \mathrm{~mm}$. Z-scores for weight, length and head circumference are then calculated with the EUROGROWTH software (http://www.euro-growth.org/). Body composition is assessed using an air displacement plethysmography system (PEA POD Infant Body Composition System, COSMED- USA). A detailed description of the PEA POD's physical design, operating principles, validation and measurement procedures is provided elsewhere [38,39]. The PEA POD assesses fat mass and fat free mass by direct measurements of body mass and volume and the application of classic densitometric principles. Infants are measured in the PEA POD naked. Each PEA POD test takes about $3 \mathrm{~min}$ to complete. Subject volume is measured in an enclosed chamber by applying gas laws that relate pressure changes to volumes of air in the chamber. Body density is then computed from the measured body mass and volume, and inserted into a standard formula for estimating the percentage of total body fat mass according to a 2-compartment model. The intra-observer coefficient of variation for the percentage of fat mass estimates is $0.3 \%$.

\section{Sample size, power and statistical analysis}

In order to detect a difference of $20 \%$ with a standard deviation of 0.3 in the erythrocyte membrane omega-3 fatty acids levels between infants receiving formula $\mathrm{A}$ and infants receiving formula $\mathrm{B}$ or formula $\mathrm{C}$, at a power of $90 \%$ and with an alpha error of $5 \%$, a total of 23 infants per group is necessary. Considering a drop out rate of about $10 \%$, a total of 75 formula fed infants, with 25 infants for each group, are included. Twenty-five exclusively breastfed infants constitute the reference group.

All statistical analyses are performed on an intention to treat basis. In addition, alternative per protocol analyses are performed, excluding all infants who are not fed according to the protocol study. Statistical analysis is performed by Soladis, Lyon, France. For statistical analysis a p-value $<0.05$ is considered significant (two tailed). All statistical analyses are performed using SPSS 12 (SPSS Inc., Chicago, IL, USA). Continuous variables are expressed as mean, standard deviation, minimum, maximum, median, quartiles.

The differences among groups in erythrocyte omega-3 FA levels, plasma lipid profile and IGF-1 levels are assessed using an analysis of covariance with sex as covariate. Differences among and within groups in repeated measurements of plasma FA levels, of growth parameters and body composition, are analyzed with an analysis of variance.

As the breastfed infants are not randomized, no statistical analysis is performed to compare the breastfed group with any of the formula feeding groups.

\section{Adverse events and serious adverse events}

Adverse events (AEs) are assessed based on clinical observation of the investigators or inquires to the parents. All AEs are recorded in adverse event forms and are evaluated by the investigator for causality for the relationship to the study feeding and for severity. An AE is defined as any event, that is not consistent with the information provided in the consent form or can reasonably be expected to accompany the natural history and progression of the subject's condition throughout the study. AEs are considered as serious (SAEs) if they are fatal or life-threatening, require hospitalization or surgical intervention, result in persistent or significant disability/incapacity or are considered to be medically relevant by the investigator. All other AEs are categorized as non-serious.

\section{Discussion}

Human milk represents the optimal nutrition for infants and ensures optimal growth and development. LCPUFA, which are provided through breast milk, play a critical role in the development of neuronal and visual functions. Hence, the achievement of an appropriate neonatal LC-PUFA status represents an important goal in neonatal nutrition, especially when breastfeeding is 
not possible $[5,7,8]$. Formula fed infants have been demonstrated to exhibit lower cerebral and erythrocyte LC-PUFA levels as compared to breast fed infants when consuming an unsupplemented formula or a formula supplemented only with EFA $[22,23]$. On the contrary, infants receiving a formula supplemented with DHA and AA show higher erythrocyte membrane omega-3 concentrations than infants fed an unsupplemented formula [24]. Recent experimental data indicate that a dairy fat blend providing as little as $1.5 \%$ ALA is superior to the plant oil blend for increasing brain DHA, even when the recommended DHA and AA levels are exogenously provided, suggesting that the endogenous synthesis of $n-3$ LC-PUFA synthesis can be enhanced [35]. Formula fed infants could then benefit from the supplementation of formula with dairy lipids and plant oils by attaining higher erythrocyte membrane omega-3 FA content than infants receiving a formula supplemented with plant oils or plants oils enriched with LC-PUFA. The achievement of optimal DHA levels in formula fed infants could also be associated with improved neurocognitive and visual functions, as previously underlined by other studies [2527]. Not only the erythrocyte membrane omega-3 FA levels will be investigated but also the other erythrocyte membrane FA levels, the plasma lipid profile, the insulin growth factor 1, the formula tolerance, the growth and body composition. Gaining insight in the effects of the supplementation of a formula with dairy lipids and plant oils in healthy full-term infants could improve the fat content, composition and structure of infant formulas, making them closer to human milk.

\section{Abbreviations}

AE: Adverse event; ALA: Alpha linolenic acid; AA: Arachidonic acid; DHA: Docosahexaenoic acid; DPA: Docosapentaenoic acid; EFA: Essential fatty acid; EPA: Eicosapentaenoic acid; FA: Fatty acid; IGF1: Insulin-like growth factor 1; LA: Linoleic acid; LC-PUFA: Long chain polyunsaturated fatty acid; SAE: Serious adverse event.

\section{Competing interests}

Lactalis Nutrition Santé, Torcé, France for the financial support and for providing the study formulas.

\section{Authors' contributions \\ Paola Roggero, Pascale Le Ruyet and Fabio Mosca formulated the research question and wrote the study protocol. Daniela Morniroli, Amandine Ligneul contributed to the development of the protocol. Francesca Garbarino gave advice on statistical analysis. Charlotte Baudry, Maria Lorella Giannì, Paola Roggero wrote the draft of the manuscript and the other authors reviewed the manuscript. All authors approved the final version of the manuscript.}

\footnotetext{
Author details

${ }^{1}$ Neonatal Intensive Care Unit (NICU), Department of Clinical Science and Comunity Health, Fondazione IRCCS "Ca' Granda" Ospedale Maggiore Policlinico, University of Milan, Milan, Italy. ${ }^{2}$ Lactalis Recherche et Développement, 8 Fromy - CS 60082, 35240, Retiers, France.
}

Received: 10 August 2012 Accepted: 11 October 2012

Published: 17 October 2012

\section{References}

1. Michaelsen KF, Weaver L, Branca F, Robertson A: Feeding and nutrition of infants and young children. European Series $N^{\circ} 87$ th edition. Copenhagen: WHO Regional Publications; 2000.

2. American Academy of Pediatrics Section on Breastfeeding: Breastfeeding and the use of human milk. Pediatrics 2005, 115:496-506.

3. ESPGHAN Committee on Nutrition, Agostoni C, Braegger C, Decsi T, Kolacek S, Koletzko B, Michaelsen KF, Mihatsch W, Moreno LA, Puntis J, Shamir R, Szajewska H, Turck D, van Goudoever J: Breast-feeding: A commentary by the ESPGHAN Committee on Nutrition. J Pediatr Gastroenterol Nutr 2009, 49:112-125

4. Jensen RG, Hagerty MM, McMahon KE: Lipids of human milk and infant formulas: a review. Am J Clin Nutr 1978, 31:990-1016.

5. Innis SM: Dietary (n-3) fatty acids and brain development. J Nutr 2007, 137:855-859.

6. Percy P, Percy A, Vilbersson G, Mansson JE: Polyunsaturated fatty acid accretion in first- and second-trimester human fetal brain: lack of correlation with levels in paired placental samples. Biochem Mol Med 1996, 59:38-43.

7. Clandinin MT, Chapell JE, Leong S, Heim T, Swyer PR, Chance PW: Intrauterine fatty acid accretion rates in human brain: implications for fatty acid requirements. Early Hum Dev 1980, 4:121-129.

8. Clandinin MT, Chappell JE, Leong S, Heim T, Swyer PR, Chance GW: Extrauterine fatty acid accretion in infant brain: implications for fatty acid requirements. Early Hum Dev 1980, 4:131-138.

9. Martinez M, Mougan I: Fatty acid composition of human brain phospholipids during normal development. J Neurochem 1998, 71:2528-2533.

10. Martinez M: Tissue levels of polyunsaturated fatty acids during early human development. J Pediatr 1992, 120(Suppl 4):129-138.

11. Lauritzen L, Hansen HS, Jorgensen MH, Michaelsen KF: The essentiality of long chain $\mathrm{n}-3$ fatty acids in relation to development and function of the brain and retina. Prog Lipid Res 2001, 40:1-94.

12. Diau GY, Hsieh A, Sarkadi-Nagy E, Wijendran V, Nathanielsz P, Brenna JT: The influence of long chain polyunsaturate supplementation on docosahexaenoic acid and arachidonic acid in baboon neonate central nervous system. BMC Med 2005, 3:11.

13. McCann JC, Ames BN: Is docosahexaenoic acid, a n-3 long-chain polyunsaturated fatty acid, required for development of normal brain function? An overview of evidence from cognitive and behavioral tests in humans and animals. Am J Clin Nutr 2005, 82:281-295.

14. Arterburn $L M$, Hall EB, Oken H: Distribution, interconversion, and dose response of $\mathrm{n}-3$ fatty acids in humans. Am J Clin Nutr 2006, 83(Suppl 6):1467-1476.

15. Hadders-Algra M: Effect of long-chain polyunsaturated fatty acid supplementation on neurodevelopmental outcome in full-term infants. Nutrients 2010, 2:790-804.

16. De Lucchi C, Pita ML, Faus MJ, Periago JL, Gil A: Changes in the fatty acid composition of plasma and red blood cell membrane during the first hours of life in human neonates. Early Hum Dev 1987, 15:85-93.

17. Larque $\mathrm{E}$, Demmelmair $\mathrm{H}$, Koletzko B: Perinatal supply and metabolism of long-chain polyunsaturated fatty acids: importance for the early development of the nervous system. Ann N Y Acad Sci 2002, 967:299-310.

18. Babin F, Limasset B, Descomps B, Rieu D, de Paulet FM, de Paulet AC: Nervonic acid in red blood cell sphingomyelin in premature infants: an index of myelin maturation. Lipids 1993, 28:627-630.

19. Fekete K, Marosvölgyi T, Jakobik V, Decsi T: Methods of assessment of n-3 long-chain polyunsaturated fatty acid status in humans: a systematic review. Am J Clin Nutr 2009, 89:2070S-2084S.

20. Lucas A, Morley R, Cole TJ, Lister G, Leeson-Payne C: Breast milk and subsequent intelligence quotient in children born preterm. Lancet 1992, 339:261-264.

21. Anderson JW, Johnstone BM, Remley DT: Breast-feeding and cognitive development: a meta-analysis. Am J Clin Nutr 1999, 70:525-535.

22. Makrides M, Simmer K, Goggin M, Gibson RA: Erythrocyte docosahexaenoic acid correlates with the visual response of healthy, term infants. Pediatr Res 1993, 33:425-427.

23. Farquharson J, Jamieson EC, Abbasi KA, Patrick WJ, Logan RW, Cockburn F Effect of diet on the fatty acid composition of the major phospholipids of infant cerebral cortex. Arch Dis Child 1995, 72:198-203. 
24. Miller MR, Seifert J, Szabo NJ, Clare-Salzler M, Rewers M, Norris JM: Erythrocyte membrane fatty acid content in infants consuming formulas supplemented with docosahexaenoic acid (DHA) and arachidonic acid (ARA): an observational study. Matern Child Nutr 2010, 6:338-346.

25. Koletzko B, Lien E, Agostoni C, Böhles H, Campoy C, Cetin I, Decsi T, Dudenhausen JW, Dupont C, Forsyth S, Hoesli I, Holzgreve W, Lapillonne A Putet G, Secher NJ, Symonds M, Szajewska H, Willatts P: Uauy R; World Association of Perinatal Medicine Dietary Guidelines Working Group: The roles of long-chain polyunsaturated fatty acids in pregnancy, lactation and infancy: review of current knowledge and consensus recommendations. J Perinat Med 2008, 36:5-14.

26. SanGiovanni JP, Berkey CS, Dwyer JT, Colditz G: Dietary essential fatty acids, long-chain polyunsaturated fatty acids, and visual resolution acuity in healthy full-term infants: a systematic review. Early Hum Dev 2000, 57:165-188.

27. Campoy C, Escolano-Margarit MV, Anjos T, Szajewska H, Uauy R: Omega 3 fatty acids on child growth, visual acuity and neurodevelopment. Br J Nutr 2012, 107(Suppl 2):85-106.

28. Courville $\mathrm{AB}$, Harel O, Lammi-Keefe CJ: Consumption of a DHA-containing functional food during pregnancy is associated with lower infant ponderal index and cord plasma insulin concentration. Br J Nutr 2011, 27:1-5.

29. Koletzko B, Bremer HJ: Fat content and fatty acid composition of infant formulas. Acta Paediatr Scand 1989, 78:513-521.

30. Michalski MC, Ollivon M, Briard V, Leconte N, Lopez C: Native fat globules of different sizes selected from raw milk: thermal and structural behavior. Chem Phys Lipids 2004, 132:247-261.

31. Berger $A$, Fleith M, Crozier G: Nutritional implications of replacing bovine milk fat with vegetable oil in infant formulas. J Pediatr Gastroenterol Nutr 2000, 30:115-130.

32. Jensen $\mathrm{RG}$, Ferris $\mathrm{AM}$, Lammi-Keefe $\mathrm{CJ}$ : Lipids in human milk and infant formulas. Annu Rev Nutr 1992, 12:417-441.

33. Jensen RG, Ferris AM, Lammi-Keefe CJ: The composition of milk fat J Dairy Sci 1991, 74:3228-3243.

34. Dabadie H, Peuchant E, Bernard M, LeRuyet P, Mendy F: Moderate intake of myristic acid in sn-2 position has beneficial lipidic effects and enhances DHA of cholesteryl esters in an interventional study. J Nutr Biochem 2005, 16:375-382.

35. Du Q, Martin JC, Agnani G, Pages N, Leruyet P, Carayon P, Delplanque B: Dairy fat blends high in a-linolenic acid are superior to $n-3$ fatty-acidenriched palm oil blends for increasing DHA levels in the brains of young rats. J Nutr Biochem 2012; . Epub ahead of print.

36. Kunz B, Oranje AP, Labrèze L, Stalder JF, Ring J, Taïeb A: Clinical validation and guidelines for the SCORAD index: consensus report of the European Task Force on Atopic Dermatitis. Dermatology 1997, 195:10-19.

37. World Health Organization Multicentre Growth Reference Study Group.WHO Child Growth Standards: length/height-for age, weight-for-age, weight forlength, weight-for-height and body mass index for-age: methods and development; 2006. Available: http://www.euro-growth.org/.

38. Ma GS, Yao M, Liu Y, Lin A, Zou H, Urlando A, Wong WW, Nommsen-Rivers L, Dewey KG: Validation of a new pediatric air displacement plethysmograph for assessing body composition in infants. Am I Clin Nutr 2004, 79:653-660.

39. Roggero P, Giannì ML, Amato O, Piemontese P, Morniroli D, Wong WW, Mosca F: Evaluation of air-displacement plethysmography for body composition assessment in preterm infants. Pediatr Res 2012, 72:316-320.

doi:10.1186/1471-2431-12-164

Cite this article as: Gianni et al:: The influence of a formula supplemented with dairy lipids and plant oils on the erythrocyte membrane omega- 3 fatty acid profile in healthy full-term infants: double-blind randomized controlled trial. BMC Pediatrics 2012 12:164.

\section{Submit your next manuscript to BioMed Central and take full advantage of:}

- Convenient online submission

- Thorough peer review

- No space constraints or color figure charges

- Immediate publication on acceptance

- Inclusion in PubMed, CAS, Scopus and Google Scholar

- Research which is freely available for redistribution

Submit your manuscript at www.biomedcentral.com/submit
Ciomed Central 